\title{
Evaluación de campo de una prueba rápida para el diagnóstico de malaria
}

\author{
Nohora M. Mendoza ${ }^{1}$, Roberto Montoya ${ }^{2}$, Marisol García ${ }^{1}$, Julio C. Padilla ${ }^{3}$, \\ Leslie O. Bruzón ${ }^{4}$, Ethel Mendoza ${ }^{4}$, Alexandra Porras ${ }^{5}$ \\ 1 Laboratorio de Parasitología, Instituto Nacional de Salud, Bogotá, D.C., Colombia. \\ 2 Oficina de Epidemiología, Ministerio de Salud, Bogotá, D.C., Colombia. \\ ${ }^{3}$ Grupo Control de Vectores, Ministerio de Salud, Bogotá, D.C., Colombia. \\ ${ }^{4}$ Secretaría Seccional de Salud de La Guajira, Riohacha, La Guajira, Colombia. \\ ${ }^{5}$ Centro de Control de Enfermedades, Instituto Nacional de Salud, Bogotá, D.C., Colombia.
}

Entre noviembre de 1999 y abril de 2000 se presentó una epidemia de malaria en el departamento de La Guajira que alcanzó 5.687 casos, 3.401 causados por Plasmodium falciparum, 2.256 por Plasmodium vivax y 30 casos diagnosticados como infección mixta. Dadas las características propias del departamento de La Guajira y de la transmisión de malaria allí, se consideró que existian las condiciones favorables para usar una prueba rápida de diagnóstico de campo. En este estudio se evaluó y comparó el diagnóstico por inmunocromatografía rápida con la gota gruesa como estándar de oro en los municipios de Dibulla, Manaure y Riohacha. La conducta con el paciente se basó en el resultado de la prueba rápida. Sin embargo, los resultados obtenidos en la gota gruesa se tuvieron en cuenta para modificar la conducta inicial con el paciente, en caso de que los resultados de las pruebas fueran discordantes. Se procesaron 231 muestras sanguíneas obtenidas por punción capilar. La sensibilidad y la especificidad generales del método inmunocromatográfico fueron de $98,7 \%$ y $99,3 \%$, respectivamente, en tanto que para $P$. falciparum fueron de $98,1 \%$ y $76,9 \%$, y para $P$. vivax de $90,9 \%$ y $100 \%$. El índice de concordancia fue 0,98 ; el valor predictivo positivo, $98,7 \%$, y el valor predictivo negativo, $99,3 \%$. Los resultados obtenidos sugieren que la prueba inmunocromatográfica (OptiMal ${ }^{\circledR}$ ) es una alternativa adecuada para realizar el diagnóstico de malaria en lugares donde no se realiza el diagnóstico microscópico.

Palabras clave: malaria, prueba de diagnóstico rápido, OptiMAL ${ }^{\circledR}$.

\section{Evaluation of a rapid diagnostic field test for malaria}

Between November 1999 and April 2000, a malaria outbreak occurred in the state of La Guajira, Colombia; 5,687 cases were diagnosed $-3,401$ as $P$. falciparum, 2,256 as $P$. vivax and 30 as mixed infections. The conditions of location and case number were considered favorable for the application and evaluation of a rapid diagnostic field test. Malaria diagnosis obtained by the rapid immunocromatographic test (OptiMAL ${ }^{\oplus}$ ) and thick blood smear were compared in the municipalities of Dibulla, Manaure and Riohacha. The treatment of each patient was based on the rapid test result, but whenever the two methods disagreed, the thick bood smear results were given priority in decisions to modify the initial treatment. Two hundred thirty-one finger-prick blood samples were processed. The general sensitivity and specificity of OptiMAL ${ }^{\circledR}$ were $98.7 \%$ and $99.3 \%$, respectively. The sensitivity and specificity for $P$. falciparum were $98.1 \%$ and $76.9 \%$. The sensitivity and specificity for $P$. vivax were $90.9 \%$ and $100 \%$. The concordance index was 0.98 , the positive predictive value, $98.7 \%$ and the negative predictive value, $99.3 \%$. These results suggest that the rapid immunocromatographic test (OptiMAL $\left.{ }^{\circledR}\right)$ is a reliable alternative for malaria diagnosis under circumstances where microscopical diagnosis is not possible.

Key words: malaria, rapid diagnosis test, OptiMAL ${ }^{\oplus}$. 
La malaria continúa siendo un grave problema de salud pública en Colombia y se estima que más de 5 millones de personas viven en áreas de riesgo. La incidencia anual de malaria ha venido en aumento; en la década de los 60 era alrededor de 1 caso por 1.000 hab y en años recientes ha aumentado a 7-8/1.000 hab debido a los problemas administrativos y financieros de los programas de salud, al desarrollo de resistencia del parásito a los antimaláricos y del vector a los insecticidas, a las condiciones precarias de vida, los conflictos sociales y los factores ambientales (1).

El diagnóstico de malaria se realiza con base en tres aspectos: diagnóstico clínico, nexo epidemiológico y diagnóstico por el laboratorio. Este último debe aplicarse en todos los casos probables de malaria para evitar el uso irracional de los medicamentos. Sin embargo, cuando se desea realizar el examen parasitológico utilizando la microscopía se necesita personal entrenado, infraestructura adecuada y un microscopio con buena óptica. Por otra parte, se han desarrollado pruebas de diagnóstico rápido (rapid diagnostic techniques, RDT) muy útiles para brindar atención médica en zonas periféricas, donde no existen facilidades técnicas.

Estas pruebas son métodos de inmunocromatografía para la captura de antígenos de Plasmodium. Los antígenos blanco más utilizados son la proteína II, rica en histidina (HRP-II), específica para la identificación de Plasmodium falciparum y la lactato deshidrogenasa parasitaria (pLDH), la cual diferencia entre malaria por $P$. falciparum y aquellas ocasionadas por un Plasmodium diferente a $P$. falciparum. Además, existen otros antígenos inespecíficos denominados panmaláricos, los cuales se utilizan en combinación con el antígeno HRP-II (2).

El antígeno pLDH es producido por las cuatro especies de Plasmodium que infectan al hombre, en estadios viables tanto sexuados como asexuados, lo cual permite que los trabajadores

\section{Correspondencia:}

Nohora Marcela Mendoza

mmendoza@hemagogus.ins.gov.co

Recibido: 07/02/01; aceptado: 06/07/01 de salud realicen diagnósticos de campo y suministren tratamientos oportunos (3).

Durante la epidemia de La Guajira, se presentó un escenario propicio para el uso de una técnica rápida (OptiMAL ${ }^{\circledR}$ ), que fuera costo/efectiva y de metodología e interpretación sencillas, ya que la epidemia estaba bajo la influencia de los siguientes factores: más del $50 \%$ de la población vivía en áreas rurales; la población nativa se consideró altamente susceptible a la malaria; la difícil accesibilidad a los puestos de atención médica; la ausencia de una red de microscopía para el diagnóstico de malaria (no existían suficientes microscopios ni personal entrenado para la lectura e interpretación de la gota gruesa) y la escasez de medicamentos antimaláricos.

El presente estudio evaluó una prueba inmunocromatográfica para el diagnóstico rápido de malaria en la epidemia de La Guajira, Colombia.

\section{Materiales y métodos}

\section{Tipo de estudio}

El estudio se basó en una evaluación de tecnología diagnóstica; se usó una muestra de corte transversal para calcular sensibilidad, la especificidad y los valores predictivos positivos y negativos. Los resultados de los dos métodos se procesaron y registraron independientemente.

\section{Sitio de estudio}

El estudio se efectuó en La Guajira, Colombia, en los municipios de Dibulla, Manaure y Riohacha. El muestreo se realizó durante las semanas epidemiológicas 10 y 11 del 2000.

\section{Tamaño de la muestra}

Se determinó un tamaño de muestra de 114 casos probables de malaria, teniendo en cuenta que la técnica OptiMAL ${ }^{\circledR}$ tiene una eficiencia de $90 \pm 5 \%$.

\section{Criterios de inclusión}

Se incluyeron en el estudio todos aquellos pacientes que cumplían con la definición de caso probable de malaria y que, además, residian en los municipios de Manaure, Riohacha o Dibulla o que consultaron a los servicios de salud pública de los mismos municipios; también, se incluyeron 
los contactos de los pacientes clasificados como casos probables de malaria.

Se definió como caso probable de malaria a toda persona residente o procedente de una zona endémica de malaria con fiebre elevada, escalofríos, sudor, mialgias o cefalea.

\section{Detección de casos}

Se realizaron búsquedas activas y se ubicaron los pacientes febriles que fueran compatibles con la definición de caso probable de malaria y sus contactos, ranchería a rancheria, en los municipios escogidos.

Por otra parte, se realizó búsqueda pasiva entre los pacientes que consultaron por sintomatología febril compatible con la definición de caso probable de malaria en los servicios de salud pública de los municipios de Dibulla, Manaure y Riohacha.

\section{Definiciones operativas}

Toma de muestra: las muestras sanguíneas se tomaron por punción capilar de todas las personas que satisfacian el criterio de caso probable de malaria y de los posibles contactos (personas que habitaban en la misma vivienda). Después de limpiar la primera gota de sangre obtenida de la punción, se realizó la toma de la muestra, primero para la prueba rápida y, luego, para la gota gruesa. Las pruebas rápidas se efectuaron en el campo, mientras que las gotas gruesas se transportaron el mismo día al Laboratorio Departamental de La Guajira para su coloración y análisis por parte de personal especializado.

Examen de gota gruesa: de acuerdo con los procedimientos estandarizados por el Instituto Nacional de Salud, la gota gruesa se tiñó con la coloración de Romanowsky y se consideró como la prueba diagnóstica de oro. La parasitemia se estimó por medio del recuento de las formas parasitarias frente a 100 leucocitos y se tomó como constante leucocitaria 8.000 leucocitos/ $\mathrm{mm}^{3}$.

Prueba rápida inmunocromatográfica $\left(O p t i M A L^{\circledR}\right)$ : la prueba rápida para el diagnóstico de malaria se fundamenta en la detección específica de la actividad de la enzima lactato deshidrogenasa de Plasmodium. Todas las pruebas fueron realizadas por personal técnico asesorado constantemente por profesionales de la salud, siguiendo la siguiente metodología:

1. Registrar los datos completos del paciente 0 de la ficha epidemiológica utilizada de rutina para el diagnóstico de malaria.

2. Identificar dos láminas y una tira reactiva por paciente.

3. Colocar en la gradilla de la prueba rápida un pozo de conjugado (rosado) y un pozo para los lavados (incoloro).

4. Adicionar una gota de solución tampón en el pozo del conjugado y cuatro gotas al de lavado y esperar un minuto.

5. Limpiar asépticamente la superficie del dedo para puncionar con una lanceta nueva; limpiar la primera gota de sangre.

6. Aspirar una gota de sangre con la pipeta. Es importante presionar bien la pipeta y no dejar burbujas en la misma. Para facilitar la toma de la muestra, esta gota debe ser de buen tamaño.

7. Dispensar la gota de sangre en el pozo de conjugado. Mezclar suavemente con la misma pipeta, teniendo cuidado de no aspirar el contenido del pozo, para lo cual se puede realizar el procedimiento con la pipeta presionada o con el extremo sellado de la pipeta. Incubar durante un minuto. Durante este tiempo se realiza la toma de muestra de las gotas gruesas.

8. Colocar verticalmente la tira reactiva dentro del pozo del conjugado. Siempre se debe tomar la tira reactiva por el extremo que viene para identificar la muestra. Incubar durante 15 minutos.

9. Transferir la tira reactiva al pozo de lavado durante 10 minutos.

10. Realizar la lectura.

Criterios de interpretación: se siguieron todas las recomendaciones de la casa fabricante (figura 1). Adicionalmente, cuando se presentó reacción muy tenue en las dos bandas correspondientes a Plasmodium spp. y P. falciparum se tuvo en cuenta el siguiente criterio: 
- Reacción negativa: cuando existe reacción en la banda de control y las otras dos bandas tienen reacción muy tenue de la misma intensidad.

- Reacción positiva: cuando, además de la banda de control, se observó que la banda para la identificación de Plasmodium spp. fue más definida que la banda específica para $P$. falciparum, a pesar de lo tenue de la reacción.

Control de calidad: todas las medidas para el control de calidad de la prueba fueron establecidas y ejecutadas por personal entrenado del Laboratorio Nacional de Referencia del Instituto Nacional de Salud a todo el material examinado (láminas y tiras reactivas). Las lecturas de las gotas gruesas obtenidas en el control de calidad se utilizaron para establecer las verdaderas muestras positivas y negativas.

La información obtenida del estudio se almacenó y analizó en una base de datos de Epilnfo 6,04. La población estudiada se estratificó por grupos de edad y a la prueba rápida se le determinó la sensibilidad, la especificidad, el índice kappa y los valores predictivos.

\section{Resultados}

Se estudiaron 231 personas clasificadas como casos probables de malaria; 78 muestras fueron positivas $(33,7 \%)$ y 153 negativas $(66,23 \%)$ por los dos métodos. Mediante la gota gruesa, 22 muestras dieron resultado positivo para $P$. vivax, 51 para $P$. falciparum y 4 para infección mixta, mientras que por la prueba rápida, 20 muestras fueron positivas para $P$. vivax y 58 para $P$. falciparum, de las cuales 4 se diagnosticaron como infección mixta por gota gruesa.

Del total de muestras, 117 se obtuvieron de mujeres $(50,6 \%)$ y 114 de hombres $(49,4 \%)$; el $63 \%$ de los casos positivos se encontró en el grupo de edad de 15 a 45 años.

Dentro de la evaluación de la prueba rápida, se encontró un falso positivo y un falso negativo para $P$. falciparum. El porcentaje de positividad del total de muestras examinadas fue de $33,8 \%(78 / 231)$ y el municipio con mayor número de muestras fue Riohacha con 201 (cuadro 1).

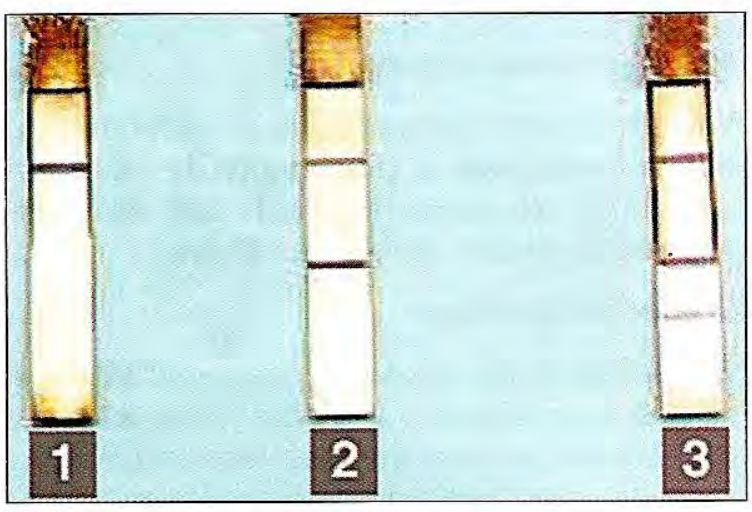

Figura 1. Reacción de la tira reactiva OptiMAL®.

1. Reacción de color solamente en la banda control: indica ausencia de Plasmodium o presencia de Plasmodium no viable.

2. Reacción de color en la banda control y en la banda de Plasmodium spp: indica la presencia de un Plasmodium diferente a $P$. falciparum; de acuerdo con el perfil epidemiológico de la zona, se concluye que es positiva para $P$. vivax.

3. Reacción de color en la banda control y en las dos bandas que indentifican Plasmodium spp y $P$. falciparum, respectivamente; reacción positiva para $P$. falciparum.

Del total de muestras positivas, el $66 \%$ fue por $P$. falciparum, hecho confirmado por la gota gruesa. Los resultados del diagnóstico por las dos técnicas se observan en el cuadro 2.

En 34 de las muestras positivas y concordantes por los dos métodos para $P$. falciparum se encontraron formas asexuadas, en 10, formas sexuadas y 7 presentaron las dos formas.

La sensibilidad general de la prueba fue de $98,7 \%$ con un intervalo de confianza de $95 \%$ (IC 95\%): 92,5-99,9 y una especificidad general de $99,3 \%$ con IC 95\%: $(95,9-100,0)$. La sensibilidad para $P$. falciparum fue de $98,1 \%$ con IC 95\% $(88,4-99,9)$; para $P$. vivax, de $90,9 \%$ con IC $95 \%$ : $(69,4-98,4)$, mientras que la especificidad para $P$. falciparum fue de $76,9 \%$ con IC $95 \%$ : $(55,9-90,2)$ y para $P$.

Cuadro 1. Resultados de la prueba rápida en los municipios estudiados.

\begin{tabular}{lrccc}
\hline Resultados & \multicolumn{3}{c}{ Municipio } & Total \\
\cline { 2 - 4 } & Dibulla & Manaure & Riohacha & \\
\hline Negativo & 19 & 2 & 132 & 153 \\
Positivo & 6 & 3 & 69 & 78 \\
Total & 25 & 5 & 201 & 231 \\
\hline
\end{tabular}


Cuadro 2. Comparación del diagnóstico obtenido por OptiMAL ${ }^{\oplus}$ y gota gruesa.

\begin{tabular}{|c|c|c|c|c|c|}
\hline \multirow[b]{2}{*}{ OptiMaL } & \multicolumn{4}{|c|}{ Gota gruesa } & \multirow[b]{2}{*}{ Total } \\
\hline & P. vivax & P. falciparum & Infección mixta & Negativo & \\
\hline$P$ vivax & 20 & 0 & 0 & 0 & 20 \\
\hline P. falciparum & 2 & 51 & 4 & 1 & 58 \\
\hline Infección mixta & 0 & 0 & 0 & 0 & 0 \\
\hline Negativo & 0 & 1 & 0 & 152 & 153 \\
\hline Total & 22 & 52 & 4 & 153 & 231 \\
\hline
\end{tabular}

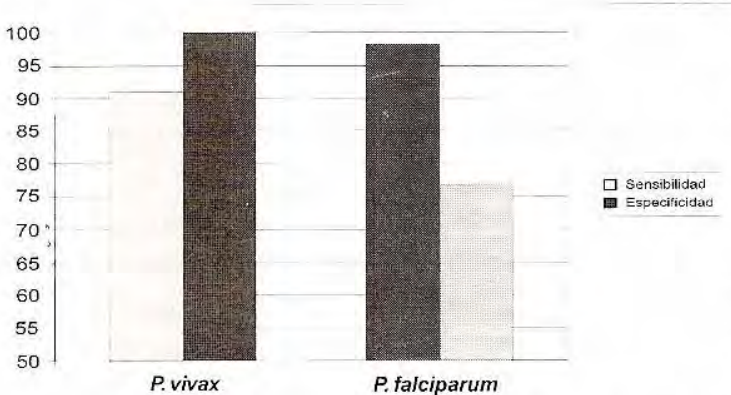

Figura 2. Sensibilidad y especificidad de la prueba rápida (OptiMAL ${ }^{\oplus}$ ) para el diagnóstico de $P$. vivax y $P$. falciparum.

vivax fue del $100 \%$ con IC 95\%: $(92,0-100,0)$ (figura 2).

Por otra parte, la prueba inmunocromatográfica presentó un índice de concordancia (kappa) de 0,98 , un valor predictivo positivo de $98,7 \%$ con IC $95 \%$ : $(92,1-99,9)$ y un valor predictivo negativo de 99,3\% con IC 95\%: $(95,9-100,0)$.

La prueba rápida fue positiva en pacientes con recuentos parasitológicos bajos (13 parásitos $/ \mu \mathrm{l})$; sin embargo, la muestra que fue diagnosticada como falso negativo presentó una parasitemia de 400 parásitos/ $\mu \mathrm{l}$.

Al evaluar la dificultad de la lectura en las dos técnicas, es decir las dudas para identificar el parásito mediante la visualización microscópica o en la lectura de la banda en la prueba rápida, se encontró que 16 muestras tuvieron dificultad por gota gruesa y 15 por el OptiMAL ${ }^{\circledR}$. Solamente 2 muestras fueron comunes por los dos métodos.

Las dificultades que se presentaron en la lectura de la tira reactiva de la prueba rápida se relacionaron con la baja definición de la banda para $P$. falciparum y la confusión en la lectura cuando las dos bandas eran muy tenues. Por otra parte, las dificultades en la gota gruesa se presentaron por problemas de la coloración o en los casos de parasitemias muy bajas.

El costo por examen de la prueba rápida fue de US $\$ 2,00$ ( $\mathrm{Col} \$ 4.200$ ) y el de la gota gruesa, US $\$ 1,80(\mathrm{Col} \$ 3.800)$.

\section{Discusión}

Los resultados del diagnóstico de malaria por la prueba rápida de diagnóstico con la microscopía tradicional son altamente comparables. La especificidad y la sensibilidad general de la prueba rápida por especie se consideraron muy cercanas a las informadas en otros estudios (4-7). Sin embargo, es importante tener en cuenta que tanto la especificidad como la sensibilidad de la prueba para $P$. falciparum se calcularon sumando las cuatro infecciones mixtas a los resultados de $P$. falciparum, debido a que estas cuatro muestras dieron resultados positivos para esta especie por el método inmunocromatográfico y, en términos prácticos, el tratamiento antimalárico inicialmente suministrado cubrió la especie de Plasmodium que representó el mayor riesgo para el paciente. También se encontraron muestras que, por complementariedad de los resultados de las dos pruebas, se habrian podido diagnosticar como infecciones mixtas y que no fueron diagnosticadas como tales por ninguno de los dos métodos. En estos casos, la prueba inmunocromatográfica dio resultado positivo para $P$. falciparum y la gota gruesa para $P$. vivax. En estas muestras, el análisis del porcentaje de trofozoítos jóvenes compatibles morfológicamente con $P$. falciparum fue de $10 \%$, criterio que no es válido por microscopía para definir una asociación (8). También existe la posibilidad de que se hubiera presentado un falso positivo para $P$. falciparum, 
tal vez ocasionado por la presencia del factor reumatoideo (9). Un diagnóstico exacto de estas muestras y las que dieron resultado falso positivo o negativo hubiera sido posible mediante la aplicación de la reacción en cadena de la polimerasa (PCR).

Para 1999, se notificaron en Colombia 788 casos de infección mixta, lo cual representa el 1,1\% de los casos del país (10). Para superar la dificultad del OptiMAL ${ }^{\circledR}$ en la identificación de las infecciones mixtas y las implicaciones que este aspecto conlleva en el tratamiento del paciente, sería importante desarrollar un anticuerpo monoclonal específico para $P$. vivax en un sitio de reacción diferente a los existentes en la tira reactiva de la prueba rápida (4). Por otra parte, quedaría por estudiar la reproducibilidad de la prueba inmunocromatográfica en parasitemias inferiores a 2.000 parásitos $/ \mu \mathrm{l}$, con el fin de descartar problemas de la prueba o posibles errores de tipo técnico.

Para resolver la dificultad que se observa en las muestras con bajas parasitemias para $P$. falciparum, sería conveniente que los fabricantes del método inmunocromatográfico aumentaran la concentración del anticuerpo monoclonal específico para esta especie.

En general, dada la concordancia casi perfecta entre las dos pruebas (11), se sugiere que la prueba rápida de diagnóstico para malaria OptiMAL ${ }^{\circledR}$ es una buena alternativa para obtener diagnósticos oportunos en áreas rurales con población dispersa, de difícil acceso y que no cuentan con atención médica oportuna. La prueba rápida constituye una valiosa herramienta para los grupos extramurales, ya que los hace más eficaces, proporcionando una atención completa (diagnóstico y tratamiento ambulatorio) en un tiempo aproximado de 27 minutos. Adicionalmente, esta prueba puede apoyar el control de la malaria en aquellos lugares que no cuentan con la infraestructura ni el personal calificado para realizar el diagnóstico por microscopía y se obtiene una mayor cobertura en la población afectada. Sin embargo, es necesario proporcionar al personal técnico o profesional un entrenamiento adecuado, teniendo en cuenta la responsabilidad en el manejo de la prueba (7).
Cuando se desea ampliar la cobertura del diagnóstico de malaria a nivel municipal, en lugares en donde no exista el diagnóstico microscópico o se dificulte por problemas de orden público o por problemas de infraestuctura básica, se debe realizar un seguimiento cuidadoso del personal entrenado y ejercer control de calidad con el fin de seleccionar el personal que demuestre responsabilidad y compromiso en la atención de los pacientes.

De igual manera, en estas áreas rurales maláricas, donde no es posible contar con un diagnóstico microscópico, se acostumbra suministrar tratamiento antimalárico con base en la clínica del paciente y se omite la posibilidad de otro tipo de infección febril como las de tipo viral. Este hecho ocasiona que los medicamentos programados para un área malárica se suministren irracionalmente y se sobrestime la ocurrencia de la enfermedad. Frente a esta problemática, el OptiMAL ${ }^{\circledR}$ es una buena alternativa para atender de manera más adecuada a estas poblaciones (2).

Otra consideración importante es el costo por prueba de las dos técnicas, el cual es ligeramente superior para la prueba rápida que para la gota gruesa; este hecho es aparente, sobre todo teniendo en cuenta que para el diagnóstico inmunocromatográfico se evitan costos de infraestructura, mantenimiento de equipos y, adicionalmente, que causa un impacto directo en la morbimortalidad por malaria, ya que permite la detección oportuna de los casos. (7).

La prueba rápida tiene una gran ventaja sobre la gota gruesa y es el hecho de eliminar la incertidumbre producida por la subjetividad inherente a las técnicas microscópicas (12). No obstante, es importante tener presente los criterios de lectura recomendados en los materiales y métodos.

Como limitante de la prueba, se puede considerar que es inadecuada para su aplicación en la evaluación de pacientes con falla terapéutica por $P$. falciparum, a diferencia de lo encontrado en la literatura $(3,13)$, en la que no se tiene presente que el OptiMAL® arroja resultados positivos cuando las muestras procesadas se obtienen de pacientes que solamente presentan en la 
circulación sanguínea formas sexuadas viables, lo cual es explicable por el principio de la prueba, que consiste en detectar un metabolito parasitario. De igual manera, la prueba tiene el inconveniente de ser cualitativa, sin que se pueda determinar con ella la parasitemia del paciente infectado.

\section{Referencias}

1. Nicholls $\mathbf{S}$. Situación actual de la malaria en Colombia. Memorias. Biomédica 2001;21(Supl.1) (en prensa).

2. World Health Organization. Malaria diagnosis. New perspectives. WHO/MAL/2000.1091. Geneva: WHO; 2000 .

3. Makler MT, Palmer CJ, Ager AL. A review of practical techniques for the diagnosis of malaria. Ann Trop Med Parasitol 1998;92:419-33.

4. Quintana M, Piper R, Boling $H$, Makler M, Sherman $\mathrm{C}$, Gill E, et al. Malaria diagnosis by dipstick assay in a Honduran population with coendemic Plasmodium falciparum and Plasmodium vivax. Am J Trop Med Hyg 1988;56:868-71.

5. World Health Organization. A rapid dipstick antigen capture assay for the diagnosis of $P$. falciparum malaria. WHO/MAL/95.1072. Geneva: WHO; 1995.

6. John SM, Sudarsaman A, Sitaram U, Moody AH. Evaluation of OptiMAL, a disptick test for the diagnosis of malaria. Ann Trop Med Parasitol 1998;92:621-2.
7. Ministerio de Salud del Perú/nstituto Nacional de Salud del Perú. Evaluación de una prueba rápida inmunocromatográfica por promotores de salud para el diagnóstico de malaria en áreas rurales de la Amazonia peruana, 1999. Informe técnico. Lima, Perú: Ministerio de Salud del Perú/INS de Perú; 1999.

8. Espinal CA, Toro G. Malaria. En: Chalem F, Escandón JE, Campos J, Esguerra R, editores. Medicina Interna. $3^{\mathrm{a}}$ ed. Santa Fe de Bogotá: Impreandes-Presencia; 1997. p. 781-92.

9. Grobusch MP, Alpermann U, Schwwenke S, Jelinek T, Warhurst DC. False-positive rapid tests for malaria in patients with rheumatoid factor. Lancet 1999;353: 297.

10. Ministerio de Salud. Vigilancia intensificada en salud pública: situación de las enfermedades transmisibles, Colombia, 1999. Inf Quinc Epidemiol Nal 2000;5:1-11.

11. Kramer MS, Fenistein AR. Clinical biostatistics. LIV. The biostatistics of concordance. Clin Pharmacol Ther 1981;29:11-23.

12. Piper R, Lebras J, Wentworth L, Hunt-Cooke A, Houzé S, Chiodini P, et al. Immunocapture diagnostic assays for malaria using Plasmodium lactate dehydrogenase. Am J Trop Med Hyg 1999;60:109-18.

13. Palmer CJ, Lindo JF, Klaskala WI, Quesada JA, Kaminsky R, Baum MK, et al. Evaluation of the OptiMAL test for rapid diagnosis of Plasmodium vivax and Plasmodium falciparum malaria. J Clin Microbiol 1998; 36:203-6. 